\title{
LILIANA BASILE. CURRICULUM VITAE
}

\author{
A cura di Raffaele Trani*
}

\section{Carriera accademica}

- $\quad$ Nata a Napoli il 17 ottobre 1950.

- 1972-73: vince una borsa di studio presso la Scuola Italiana di Storia del Pensiero Economico (SISPE), diretta da Claudio Napoleoni.

- 22.7.1974: si laurea con lode in Economia e Commercio, presso la Facoltà di Economia e Commercio dell'Università Federico II di Napoli, discutendo una tesi di laurea su "Tendenze recenti dell'economia degli USA (1965-'72)", relatore: Mariano D’Antonio.

- 1974-75: vince una borsa di studio della Scuola di Sviluppo Economico (SSE) presso l'Unione delle Camere di Commercio.

- 1.5.1975-14.2.1982: è assegnista presso l'Istituto di Economia della Facoltà di Economia e Commercio dell'Università Federico II di Napoli.

- 15.2.1982-31.10.1992: è ricercatore confermato presso il Dipartimento di Scienze Economiche e Sociali della Facoltà di Economia e Commercio dell'Università Federico II di Napoli.

- 1990-91 e 1991-92: ha una supplenza di Economia Politica presso la Facoltà di Economia e Commercio dell'Università Federico II di Napoli.

- 1.11.1992-30.10.2001: è professore associato di Economia Politica (settore P01A) presso la Facoltà di Economia dell'Università Federico II di Napoli.

- Dall'1.11.2001: è professore straordinario di Economia

\footnotetext{
*Università Federico II Napoli, e-mail: rafftrani@libero.it
} 
Politica (settore P01A) presso la Facoltà di Economia dell'Università Federico II di Napoli.

- Dall'1.11.2006: è professore ordinario di Economia Politica (settore P01A) presso la Facoltà di Economia dell'Università Federico II di Napoli.

- Dall'a.a. 2004/2005 è membro del Collegio dei docenti del Dottorato di ricerca in Economia e Politica dello Sviluppo, coordinato da Carlo Panico, con sede presso l’Università Federico II di Napoli.

Soggiomi di studio

\section{Attività}

scientifica
- $\quad$ 1.10.1980-31.3.1981: Cambridge (UK), presso la Faculty of Economics, University of Cambridge, finanziato con fondi CNR ("Altri Interventi").

- 1.10-31.12.1981: Cambridge (UK), presso la Faculty of Economics, University of Cambridge, finanziato con fondi CNR ("Altri Interventi").

- 26.10-14.12.1985: Manchester (UK), presso la Faculty of Economic and Social Studies, University of Manchester, finanziato con fondi MPI.

- 24.8-13.11.1987: Cambridge (UK), presso la Faculty of Economics, University of Cambridge, finanziato con fondi CNR ("Altri Interventi").

- 2.12-21.12.1987: Cambridge (UK), presso la Faculty of Economics, University of Cambridge, finanziato con fondi MPI.

- 18.10-13.12.1990: Cambridge (UK), presso la Faculty of Economics, University of Cambridge, finanziato con fondi CNR ("Altri Interventi").

- 11.1-24.2.1991: Cambridge, Mass. (USA), presso il Department of Economics, Massachusetts Institute of Technology (MIT), finanziato con fondi CNR ("Altri Interventi").

- È stata responsabile dei progetti di ricerca MURST 60\% "Organizzazione e dimensione in alcune imprese italiane" (1992); "Ipotesi sulla razionalità e natura dell'impresa" (1993 e 1994); "Nuovi diritti di proprietà e visione contrattuale dell'impresa" (1995) e "Informazione incompleta e natura contrattuale dell'impresa" (1996).

- È stata membro dei progetti di ricerca MURST 40\% "Istituzioni, crescita e sviluppo economico" (1992 e 1993, coordinatore centrale: Sergio Parrinello; coordinatore locale: Massimo Marrelli); "Occupazione: problemi di analisi e di politica" (1994 e 1995, coordinatore centrale: Nicola Acocella; coordinatore locale: Maurizio Pugno). Di 
quest'ultimo progetto è stata coordinatrice locale nell'anno 1996.

- È stata membro del programma di ricerca nazionale cofinanziato "Contrattazione incompleta e analisi delle Istituzioni” (1998 e 1999, coordinatore nazionale: Ugo Pagano; responsabile dell'unità di ricerca locale: Massimo Marrelli).

- È responsabile di una unità di ricerca locale nel programma di ricerca nazionale "Analisi economica comparata delle Istituzioni e complessità istituzionale delle forme di governance nella prospettiva della teoria dei contratti incompleti" (richiesta di cofinanziamento, anni 2000 e 2001, coordinatore nazionale: Francesco Silva).

- È membro del "Gruppo di Studio delle Teorie e delle Politiche Economiche", costituitosi nel 1988 con finanziamento "Altri interventi" del CNR e coordinato da Neri Salvadori.

- È stata responsabile scientifico del progetto di ricerca CNR (1996) "Controllo, proprietà e organizzazione: un confronto fra settore pubblico e settore privato nell'economia italiana".

- È stata responsabile scientifico del programma di ricerca nazionale, anno 2002, "Il governo della proprietà intellettuale: incompletezza contrattuale, complementarità istituzionale e politiche alternative dell'innovazione e dello sviluppo", coordinatore nazionale prof. Ugo Pagano.

- È stata responsabile scientifico del programma di ricerca nazionale, anno 2005, "Vantaggio Istituzionale Comparato, Proprietà Intellettuale e Globalizzazione dei Mercati", coordinatore nazionale prof. Ugo Pagano.

Elenco delle pubblicazioni
- 1. "Prezzi e distribuzione in Michal Kalecki", Studi Economici, 1980, n. 12, pp. 27-56.

- 2. (in collaborazione con M. D'Antonio), "Prezzi, costi e redditi in agricoltura: un'analisi dei recenti provvedimenti comunitari”, La Questione Agraria, 1981, n. 2, pp.59-68.

- 3. (in collaborazione con N. Salvadori), "Kalecki's Pricing Theory", Journal of Post Keynesian Economics, Winter 1984-85, n. 2, pp. 249-62; ristampato in M. Blaug (ed.), Michal Kalecki (1899-1970), Edward Elgar, Aldershot, 1992; e in M. C. Sawyer (ed.), The Legacy of Michal Kalecki, Edward Elgar, Cheltenham, 1999.

- 4. "Recensione dell'articolo di M. Sebastiani sull'equilibrio di sottoccupazione nel pensiero di M. Kalecki”, Studi Economici, 1986, n. 28, pp. 143-7. 
- 5. "Causalità ed economia", Economia Politica, 1986, n. 2, pp. 233-58.

- 6. (in collaborazione con N. Salvadori), "Kalecki’s Pricing Theory: A Reply", Journal of Post Keynesian Economics, Fall 1986, pp. 159-60.

- 7. Recensione di "Herbert A. Simon, Causalità, razionalità, organizzazione, Il Mulino, Bologna, 1985", Economia Politica, 1987, n. 2, pp. 326-30.

- 8. (in collaborazione con A. Callegari), "Evoluzione demografica e struttura produttiva a Napoli secondo i dati degli ultimi censimenti”, Mezzogiorno d'Europa, 1987, n. 4, pp. 529-583; ristampato in versione inglese: "Demographic Evolution and Production Structure in Naples According to the Information of the Latest Censues", Journal of Regional Policy, 1987, n. 4, pp. 523-577.

- 9. (in collaborazione con F. Polverini), "Connessione causale e test di causalità", Quaderni di Storia dell'Economia Politica, 1987-'88, n. 3-1, pp. 185-208.

- 10. Recensione di "A. Vercelli, Keynes dopo Lucas, La Nuova Italia Scientifica, Roma, 1987”, Studi Economici, 1988, n. 36, pp.155-161.

- 11. "Uncertainty in Economic Theory: an Epistemological Perspective", Ricerche Economiche, 1989, n. 1-2, pp. 218235.

- 12. (in collaborazione con L. Cavola e M. D'Antonio), L'industria turismo, 1990, Roma, Editore SIPI.

- 13. Recensione di “M. Sebastiani (ed.), Kalecki's Relevance Today, London, Macmillan, 1989", Contributions to Political Economy, 1990, pp. 113-116.

- 14. (in collaborazione con N. Salvadori), "Kalecki's Pricing Theory Revisited", Journal of Post Keynesian Economics, Winter 1990-'91, n. 2, pp. 293-297.

- 15. (in collaborazione con P. Casavola), "L'impresa come soggetto istituzionale: efficienza e contrattazione incompleta", Note Economiche, 1991, n. 2, pp. 272-294.

- 16. (in collaborazione con N.Salvadori), "Kalecki's Pricing Theory", Michal Kalecki (1899-1970), 1992, pp. 11528, Elgar Reference Collection series. Pioneers in Economics series, vol. 39. Aldershot, U.K.: Elgar; distributed in the U.S. by Ashgate, Brookfield, Vt.

- 17. "Kalecki's Macro-dynamic Theory of Business Cycles", Studi Economici, 1992, n. 48, pp. 127-135.

- 18. (in collaborazione con N. Salvadori), "On the Existence of a Solution to Kalecki's Pricing Equations"; Journal of Post Keynesian Economics, Spring 1994, n. 3, pp. 435-438. 
- 19. (in collaborazione con P. Casavola), "The Firm as an Institution: Recent Evolution in the Contractual Perspective", Recherches Economiques de Louvain, 1994, n. 2, pp. 249-270.

- 20. "Ipotesi sulla razionalità e natura dell'impresa", in B. Jossa-U. Pagano (a cura di), Economie di mercato ed efficienza dei diritti di proprietà, 1997, Torino, Giappichelli Editore.

- 21. "Nuovi diritti di proprietà e visione neo-istituzionalista dell'impresa", Studi Economici, 1999, n. 69, pp. 51-67.

- 22. (in collaborazione con N. Salvadori), "Kalecki's Pricing Theory", The legacy of Michal Kalecki. Volume 1, 1999, pp. 316-29, Elgar Reference Collection. Intellectual Legacies in Modern Economics, vol. 5. Cheltenham, U.K. and Northampton, Mass.: Elgar; distributed by American International Distribution Corporation, Williston, $\mathrm{Vt}$.

- 23. Recensione di "H.E. Simon, Scienza economica e comportamento umano, Edizioni di Comunità, Torino, 2000", Studi Economici, 2001, 74, pp. 153-156.

- 24. "Razionalita ed efficienza nel programma di ricerca di Herbert Simon", Studi Economici, 2002, v. 57, iss. 78, pp. $115-22$.

- 25. "Incomplete Contracts and Intellectual Property Rights", Studi Economici, 2004, v. 59, iss. 84, pp. 43-58.

- 26. (in collaborazione con R. Trani), "Incomplete Contracts Modelling", Metroeconomica, July 2008, v. 59, iss. 3 , pp. 347-70.

Seminari e convegni
- 26.4.1984: seminario dal titolo "La teoria dei prezzi di M. Kalecki”, tenuto presso il Dipartimento di Scienze Economiche e Sociali, Università di Napoli.

- 26.5.1984: seminario dal titolo "Le connessioni tra la teoria dei prezzi di Kalecki e le teorie del 'costo pieno",, tenuto presso l'Istituto Economico-Finanziario della $\mathrm{Fa}$ coltà di Scienze Politiche, Università di Napoli.

- 7-9.5.1984: relazione dal titolo "Il ruolo del grado di monopolio nella teoria dei prezzi di Kalecki", presentata al convegno "Domanda effettiva, prezzi e distribuzione", Siena.

- 23-25.5.1985: relazione dal titolo "Causalità e interdipendenza nel dibattito keynesiano", presentata al convegno "Domanda effettiva, prezzi e distribuzione", Catania.

- 27.2-1.3.1986: relazione dal titolo "Il mark-up in approcci teorici alternativi", presentata al Convegno "Domanda effettiva, prezzi e distribuzione", Catania. 
- 27-2911.1987: relazione dal titolo "Connessione causale e test di causalità", presentata al Convegno "Causalità e interdipendenza nella storia dell'analisi economica”, Siena.

- 24-26.3.1988: relazione dal titolo "Inferenza causale e inferenza associazionale", presentata al convegno "Domanda effettiva, prezzi e distribuzione", Catania.

- 7-9.10.1988: relazione dal titolo "L'incertezza nella teoria economica in una prospettiva epistemologica", presentata al convegno "Domanda effettiva, prezzi e distribuzione", Siena.

- 15-17.2.1990: relazione dal titolo "Prezzi, profitto e grado di monopolio", presentata al convegno "Domanda effettiva, prezzi e distribuzione", Catania.

- 14-15.12.1990: relazione dal titolo "L'impresa come soggetto istituzionale: efficienza e contrattazione incompleta", presentata al convegno "Razionalità, istituzioni e nozione di concorrenza", Venezia.

- 15-17.3.1991: relazione dal titolo "The Firm as an Institution: Efficiency and Incomplete Contracts", presentata alla Eastern Economic Association $17^{\text {th }}$ Annual Convention, Pittsburgh, PA.

- 15-16.9.1991: relazione dal titolo "Residual Control Rights and Investment Decisions", presentata al convegno "Organizzazione della produzione e Istituzioni", Bressanone.

- 19-20.9.1991: relazione dal titolo "The Firm as an Institution: Recent Evolution in the Contractual Perspective", presentata al convegno "L'Institutionnalisme en Question", Marsiglia.

- 27-29.3.1992: relazione dal titolo "The Firm in 'the Incomplete Contracting' View", presentata alla Eastern Economic Association 18th Annual Convention, New York.

- 1-3.10.1992: relazione dal titolo "Razionalità ed efficienza dell'impresa nella visione contrattuale", presentata alla riunione del gruppo MURST 40\% "Istituzioni, crescita e sviluppo economico", Siena.

- 30.9-1.10.1993: relazione dal titolo "Ipotesi sulla razionalità e natura dell'impresa", presentata al IX Convegno AISSEC (Associazione Italiana per lo Studio dei Sistemi Economici Comparati), Milano.

- 27.10.1993: seminario dal titolo "Nuovi diritti di proprietà e visione contrattuale dell'impresa", tenuto presso il Dipartimento di Scienze Economiche e Sociali, Università "Federico II" di Napoli, nell'ambito del secondo ciclo di 
seminari dal titolo "Economia e Istituzioni: fondamenti di teoria e di politica economica", Napoli.

- 29-30.4.1994: relazione dal titolo "Le nuove teorie dei diritti proprietari”, presentata alla riunione del gruppo MURST 40\% "Occupazione: problemi di analisi e di politica", Cosenza.

- 23.5.1998: seminario dal titolo "La contrattazione incompleta come ipotesi di non-verificabilità delle norme contrattuali", tenuto presso la Facoltà di Economia dell'Università Federico II di Napoli, Napoli.

- 19.4.1999: seminario dal titolo " Privatizzazione e regolamentazione secondo l'approccio dei Nuovi Diritti di Proprietà", tenuto presso la Facoltà di Economia dell’Università Federico II di Napoli, Napoli.

\section{Altre attività}

- $\quad$ È referee per le Riviste: Metroeconomica, Post Keynesian Journal of Economics, Studi Economici e The Economic Journal.

- Dal 1984-85 al 1989-90 è stata tutor di gruppi di studio di varie nazionalità organizzati nell'ambito dei corsi biennali "Master in Economia dello Sviluppo", svolti presso l'Istituto di Studi per lo Sviluppo Economico (ISVE). 